\title{
First results from XILO: XMM-Newton Investigations in the Lambda Orionis star forming region
}

\author{
B. Stelzer ${ }^{1}$, D. Barrado y Navascues ${ }^{2}$, N. Huelamo ${ }^{2}$, \\ M. Morales-Calderon ${ }^{2}$, A. Bayo ${ }^{2}$ \\ ${ }^{1}$ INAF - Osservatorio Astronomico di Palermo, 90134 Palermo, Italy \\ email: stelzer@astropa.unipa.it \\ ${ }^{2}$ LAEFF - INTA, 28691 Villanueva de la Canada, Spain
}

The $\lambda$ Orionis star formation region $(1-6 \mathrm{Myr}, 400 \mathrm{pc})$ is a complex of star-forming clouds surrounded by a molecular ring with $\sim 5^{\circ}$ radius which was probably formed by a supernova explosion (Dolan \& Mathieu 2002). For a complete picture of star formation, believed to be determined by the supernova blast, the large-scale distribution of the pre-main sequence population in $\lambda$ Ori needs to be examined. We have embarked on a multi-wavelength study (XMM-Newton/Xray, CFHT/optical, Spitzer/IR) of selected areas within this intriguing star-forming complex that enables us to identify young stars and brown dwarfs. Our study comprises various areas within the cloud complex as shown in Fig.1. This data set is among the most extended X-ray surveys carried out with $X M M$-Newton in a coherent star-forming environment. The XMM-Newton observations combined with optical and IR data reveal the low-mass stellar population down to $\sim 0.4 M_{\odot}$. For this mass-limited sample, our preliminary analysis confirms the anomalously low disk-fraction of the central star cluster Coll 69, the Eastern extension of its low-mass population pointing towards B 35, and the concentration of young stars in front of B 35. The analysis of the 'on-cloud field' of B 35 (white in the figure) will show if the cloud is currently forming stars. This will be crucial for determining the star-forming history in the whole $\lambda$ Ori region.

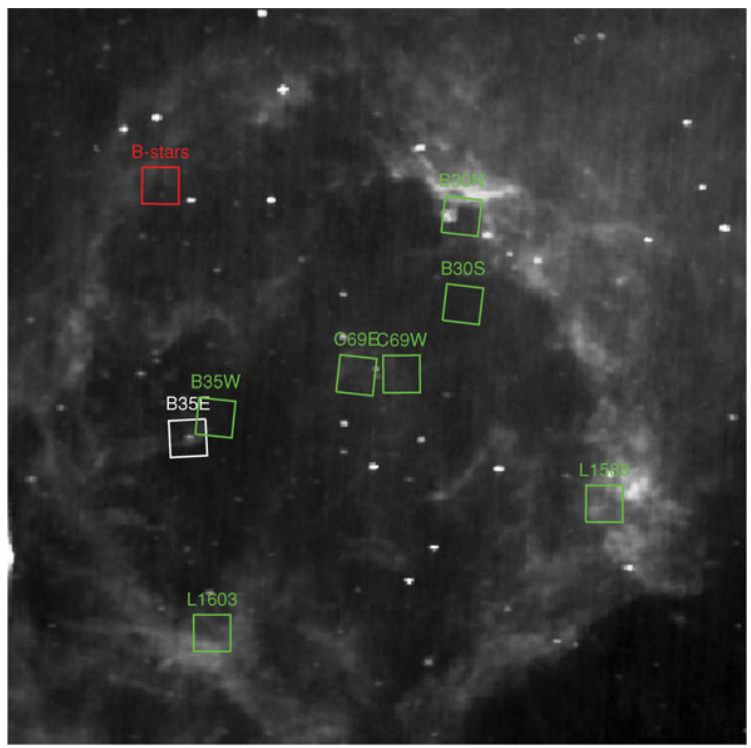

Figure 1. IRAS $100 \mu \mathrm{m}$ map of the $\lambda$ Ori region with $X M M$-Newton pointings from the XILO project overlaid. The observed areas comprise the eastern and western sides of the central star cluster Collinder 69, the dark clouds B 30 \& B 35, the 'bridge' connecting B 30 with Coll 69, the dark clouds LDN 1603 and LDN 1588 on the molecular ring, and a concentration of $\mathrm{B}$ stars near the north-east of the ring. The pointings from the original XILO project are shown in green, one observation yet to be executed is shown in red (field labelled 'B-stars'), and data added from the XMM-Newton archive are shown in white (field labelled 'B35E'). 